\title{
ORGANOFILIZAÇÃO E SILANIZAÇÃO DE ARGILA BENTONITA
}

\author{
Amanda F. Baruel ${ }^{\mathrm{a}}$, Rita C. L. Dutra ${ }^{\mathrm{a}}$, Maurício R. Baldan ${ }^{\mathrm{a}, \mathrm{c}}$, Cristina M. A. Lopes $^{\mathrm{a}, \mathrm{b}}$ e Silvana N. Cassu ${ }^{\mathrm{a}, \mathrm{b}, *}$ \\ anstituto Tecnológico da Aeronáutica, Departamento de Ciência e Tecnologia Aeroespacial, 12228-904 São José dos Campos - \\ SP, Brasil \\ 'Instituto de Aeronáutica e Espaço, Departamento de Ciência e Tecnologia Aeroespacial, 12228-904 São José dos Campos - SP, Brasil \\ 'Instituto Nacional de Pesquisas Espaciais, CP 515, 12227-010, São José dos Campos - SP, Brasil
}

Recebido em 30/06/2017; aceito em 16/10/2017; publicado na web em 04/12/2017

\begin{abstract}
ORGANOPHILIZATION AND SILANIZATION OF BENTONITE CLAY. Clays have been used in several technological areas. One of the most usual applications is their incorporation into organic materials. Nonetheless, natural clays are hydrophobic being incompatible with organic medium. Clay organophilization can be reached by several methods such as by exchange of the interlayer sodium cations by organic cations, and by silanization of the clay layers. In this study the bentonite clay was organophilized by the addition of the methoxymethyltriphenylphosphonium chloride (CTFF). Further, the organoclay was silanized with (3-glycidyloxypropyl) trimethoxysilane (GPTMS). X-ray fluorescence results, infrared and XPS spectroscopy showed the presence of phosphorus in the organophilic clay. The interlayer space, obtained by X-ray diffraction, increased in 55\% after the CTFF addition showing the cationic exchange in the clay lamellar region. After the silanization of the organoclay the interlayer space was slightly reduced from $1,78 \mathrm{~nm}$ to $1,72 \mathrm{~nm}$, indicating that the silane molecules were preferential bound in the clay edges.
\end{abstract}

Keywords: organoclay; bentonite; silane; quaternary phosponium salt.

\section{INTRODUÇÃO}

A argila é definida como um material terroso, de granulação fina, apresenta alta plasticidade em água ${ }^{1-3}$ e é formada pelo empacotamento de lamelas cristalinas..$^{1,3-5} \mathrm{~A}$ bentonita uma das argilas de maior interesse tecnológico, ${ }^{3,6,7}$ apresenta em sua configuração cátions interlamelares, que podem ser trocados por cátions orgânicos ou inorgânicos quando presentes em solução aquosa, influenciando suas propriedades físico-químicas e alterando seu espaçamento basal, essa propriedade é chamada de capacidade de troca catiônica (CTC) ${ }^{2-4,8,9} \mathrm{O}$ termo bentonita é comumente usado para designar argilas constituídas principalmente por argilomineral ou argilominerais esmectíticos, sendo o argilomineral mais frequentemente encontrado a montmorilonita. Essa classe de argilas é constituída principalmente por silicatos de alumínio e magnésio do tipo 2:1, no qual uma folha octaédrica está entre duas folhas tetraédricas. Esse silicato possui deficiência de cargas positivas em sua estrutura cristalina, resultando em excesso de cargas negativas, que é contrabalanceado por cátions interlamelares alcalinos, $\mathrm{Na}+$ ou $\mathrm{K}+$. A montmorilonita é caracterizada pela sua capacidade de troca catiônica, que permite que os cátions inorgânicos sejam substituídos por cátions orgânicos, aumentando seu caráter apolar, e possibilitando sua incorporação em meios orgânicos. ${ }^{3}$ Após a troca catiônica as argilas são denominadas argilas organofílicas ou organofilizadas, e a partir dessas é possível se obter complexos argila-compostos orgânicos. ${ }^{2,10}$ As argilas organofilizadas são mais facilmente incorporadas em sistemas orgânicos, como em cosméticos, tintas à base de solventes orgânicos, polímeros apolares e lubrificantes. A substituição de cátions de sódio por cátions orgânicos, em geral, causa um aumento da distância basal da argila, o que pode ser observado pela técnica de difração de raios-X (XRD) e, usualmente, quanto maior a cadeia orgânica do sal maior será a distância interlamelar alcançada. ${ }^{11}$ Sais quaternários de amônio são os mais comumente utilizados para esse tipo de modificação, já que podem facilmente substituir os cátions mono ou divalentes presentes na argila bentonita. ${ }^{12-17}$ Porém, os cátions quaternários de amônio não conseguem suportar temperaturas de processamento muito elevadas, ${ }^{18-21}$ sendo assim, argilas organofílizadas por meio de um sal de amônio são menos adequadas para utilização em materiais que utilizam altas temperaturas de processamento, como em polímeros contendo argilas como reforço, que são submetidos a processos de extrusão. ${ }^{20}$ $\mathrm{Na}$ literatura, a estabilidade térmica dos sais de fósforo é descrita sendo superior à apresentada por sais de amônio. ${ }^{10,20-23}$ Compostos de fósforo são utilizados em diversas aplicações, tais como retardantes de chama para papeis e têxteis, e também como absorvedores de radiação ultra violeta em materiais poliméricos. A adição de argilas organofilizadas com sais de fósforo vem sendo investigada em matrizes poliméricas visando aumentar sua resistência térmica e retardância à chama. ${ }^{22,23}$

Outro método estudado para modificar a argila é a sua silanização. ${ }^{24,25}$ Neste método é utilizado um organosilano, contendo pelo menos dois grupos reacionais, um que pode se condensar com os grupos hidroxila da argila, formando ligações covalentes entre o silano e os grupos funcionais presentes na superfície das lamelas do mineral, e outro grupo reacional que reagirá ou apresentará forte interação com o meio no qual a argila será incorporada. ${ }^{26-30} \mathrm{~A}$ reação de silanização é irreversível, diferentemente da troca catiônica, devido às ligações covalentes formadas entre a argila e o silano. ${ }^{24,25}$

Este estudo propõe a organofilização menos estudada, por meio da incorporação de um sal de fósforo à argila bentonita, visando modificar seu caráter hidrofílico. Além da organofilização, a argila foi também submetida à silanização com o objetivo de se introduzir grupos epóxidos à sua estrutura, com isso aumentando sua reatividade em relação a compostos que contenham grupos amina ou anidrido. As argilas modificadas foram caracterizadas por diversas técnicas, sendo possível se verificar tanto a substituição dos cátions de sódio por cátions do sal quaternário de fósforo, como a incorporação do silano à argila. 


\section{PARTE EXPERIMENTAL}

\section{Materiais}

A argila bentonita sódica expansível em água foi fornecida pela empresa Bentonisa do Nordeste. A capacidade de troca catiônica da argila foi determinada utilizando-se o método de azul de metileno (MB) ${ }^{31-33}$ dissolvendo-se $1 \mathrm{~g}$ de argila em $60 \mathrm{~mL}$ de água deionizada e gotejando-se uma solução de $0,15 \mathrm{~mol} \mathrm{~L}^{-1}$ de azul de metileno. A determinação da área superficial da argila também empregou o método de azul de metileno ${ }^{31-33} \mathrm{O}$ sal quaternário de fósforo utilizado para a organofilização foi o cloreto de (metoximetil) trifenil fosfônio (CTFF) (Sigma Aldrich). O silano empregado foi o (3-glicidilpropil) trimetoxisilano (GPTMS). As estruturas químicas do CTFF e GPTMS são apresentadas na Figura 1.
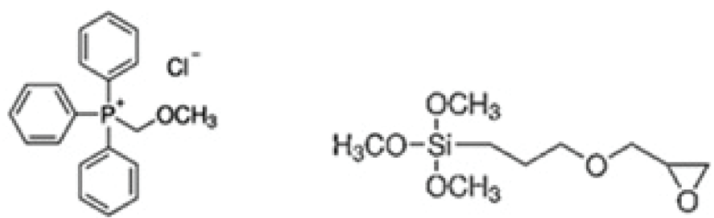

Figura 1. Estrutura química do CTFF e GPTMS ${ }^{34}$

\section{Organofilização da argila bentonita}

Para a retirada de parte das impurezas da argila, como quartzo e feldspato, a argila bentonita foi seca em estufa a $100{ }^{\circ} \mathrm{C}$ por $24 \mathrm{~h}$, e então foi passada em peneira com abertura da malha de $53 \mu \mathrm{m}$. Essa argila foi chamada de argila P.

A organofilização da argila ${ }^{4,17,21}$ foi realizada a partir de uma dispersão de cerca de $15 \mathrm{~g}$ de argila em $900 \mathrm{~mL}$ de água deionizada, que foi agitada por 20 min em agitador mecânico, marca Marconi, modelo MA 259, com hélice do tipo roseta dentada, com diâmetro de $45 \mathrm{~mm}$, e haste com $280 \mathrm{~mm}$ de comprimento, diâmetro de 3/8 pol, ambos em aço inoxidável. A velocidade empregada para obtenção da dispersão aquosa da argila foi de $700 \mathrm{rpm}$. Em seguida preparou-se uma solução de $6 \mathrm{~g}$ de CTFF, em $120 \mathrm{~mL}$ de dimetilformamida (DMF), que foi adicionada à dispersão de argila e agitada por $8 \mathrm{~h}$ a $25^{\circ} \mathrm{C}$ sob agitação a $1500 \mathrm{rpm}$. A massa de CTFF foi calculada tendo por base a capacidade de troca catiônica da argila, e considerando-se que cada molécula do CTFF poderia substituir um átomo de sódio. Após agitação, realizou-se a lavagem da argila organofilizada resultante, aproximadamente $9,5 \mathrm{~g}$, com cerca de $8 \mathrm{~L}$ de água deionizada até a completa remoção dos sais, visualizada pela adição de nitrato de prata $\left(\mathrm{AgNO}_{3}\right) 0,1 \mathrm{~mol} \mathrm{~L}^{-1}$ à água de lavagem até que não se verificou a formação de precipitado branco de cloreto de prata $(\mathrm{AgCl})$. $^{1,35}$ Realizou-se uma lavagem com $200 \mathrm{~mL}$ de tetrahidrofurano (THF) e uma última lavagem com $200 \mathrm{~mL}$ de acetona, a fim de arrastar o residual de água ainda presente na dispersão de argila. Para caracterização, a argila foi seca em estufa a vácuo, a $50{ }^{\circ} \mathrm{C}$ (temperatura suficiente para remoção da acetona e demais voláteis sob vácuo), por $24 \mathrm{~h}$, e nomeada de AO.

\section{Silanização da argila organofílica}

A silanização da argila organofilizada (AO) foi feita de acordo com a literatura, ${ }^{24,35,36}$ partindo-se de $3 \mathrm{~mL}$ de $\mathrm{AO}$ (aproximadamente $0,5 \mathrm{~g}$ ) dispersa em $20 \mathrm{~mL}$ de acetona sob agitação por $10 \mathrm{~min}$. Após agitação foi adicionado 0,0496 $\mathrm{g}$ de GPTMS e $10 \mathrm{~mL}$ de acetona (10\% em massa), mantidos sob agitação por 1 h e 40 min. Após esse período a dispesão foi submetida ao ultrassom de alta potência com amplitude de $60 \%$ e ciclo 1 por $1 \mathrm{~h}$ e $30 \mathrm{~min}$. A argila organofilizada e silanizada (AOS) foi seca em estufa a $70{ }^{\circ} \mathrm{C}$, desagregada em almofariz de ágata e, então, caracterizada.

\section{Caracterização}

As argilas foram caracterizadas por fluorescência de raios-X (XRF), difração de raios-X (XRD), termogravimetria (TGA), espectroscopia fotoeletrônica de raios-X (XPS), análise elementar CHN e espectroscopia no infravermelho com transformada de Fourier (FT-IR).

A análise por XRF foi realizada no equipamento Axios Advanced, PanAnalytical, com tubo de Rh e potência de 2,5 W. A aquisição de dados foi feita com o software Super Q Manager, sendo o resultado normalizado para $100 \%$. A amostra foi analisada na forma de pastilha prensada.

A análise de XRD foi realizada no equipamento X Pert Pro MPD da PanAnalytical, operando à tensão de $40 \mathrm{kV}$, corrente de $45 \mathrm{~mA}$, na faixa $2 \theta$ de $3^{\circ}$ até $90^{\circ}$, raios-X obtidos utilizando-se tubo de cobre e filtros de níquel e comprimento de onda do raio incidente de $0,1541 \mathrm{~nm}$. A lei de Bragg, $\lambda=2 \mathrm{dsen} \theta$, foi usada para calcular a distância interplanar basal (001).

As análises termogravimétricas foram realizadas no equipamento TG/DTA6200 SII Nanotechnology, em faixa de temperatura de $25^{\circ} \mathrm{C}$ a $1000{ }^{\circ} \mathrm{C}$, com taxa de aquecimento de $10{ }^{\circ} \mathrm{C} \mathrm{min}^{-1}$, em porta amostra de alumina com atmosfera de nitrogênio com fluxo de $100 \mathrm{~mL} \mathrm{~min}{ }^{-1}$. As análises foram realizadas em duplicata, desde que desvios da ordem de até $1 \%$ em massa fossem obtidos.

$\mathrm{A}$ avaliação dos elementos presentes nas superfícies da argila $\mathrm{Pe}$ argilas AO e AOS foi feita utilizando-se a técnica de espectroscopita fotoeletrônica de raios X (XPS), empregando-se o equipamento AXIS Ultra DLD com fonte de raios X de $\mathrm{Mg} \mathrm{K} \alpha(1253,6 \mathrm{eV})$. O XPS foi calibrado com padrão prata e a faixa de energia variou de 0 a $1200 \mathrm{eV}$. A análise por $\mathrm{CHN}$ foi realizada no equipamento $2400 \mathrm{CHNS} / \mathrm{O}$ Analyser Series II da PerkinElmer, utilizando como gás de arraste hélio 4.5 com pressão de 18 psi e como gás de combustão oxigênio 6.0 com pressão de 15 psi, faixa de massa entre 3,5 e 4,5 mg.

Os espectros de infravermelho foram obtidos utilizando-se o equipamento Spectrum One FT-IR Spectrometer-PerkinElmer, faixa espectral de 4000 a $550 \mathrm{~cm}^{-1}$, ganho 1 , resolução $4 \mathrm{~cm}^{-1}, 20$ varreduras. Os espectros foram obtidos no modo de reflexão por meio do acessório Drift ou utilizando-se o acessório de refletância total atenuada universal (UATR) aplicando-se uma força de $120 \mathrm{~N}$.

\section{RESULTADOS E DISCUSSÃO}

Inicialmente foram estimados os valores de capacidade de troca catiônica e área superficial da argila $\mathrm{P}$ pelo método de azul de metileno, obtendo-se o valor de $114 \mathrm{meq} / 100 \mathrm{~g}$ de argila para a capacidade de troca catiônica, que está de acordo com o descrito na literatura ${ }^{2,37}$ para argilas dos tipos bentonita e montmorilonita, que varia entre $80 \mathrm{meq} / 100 \mathrm{~g}$ de argila a $150 \mathrm{meq} / 100 \mathrm{~g}$ de argila. A área superficial da argila $\mathrm{P}$ foi estimada em $894,5 \mathrm{~m}^{2} \mathrm{~g}^{-1}$, estando dentro da faixa descrita ${ }^{38,39}$ para esse tipo de argila $\left(470 \mathrm{~m}^{2} \mathrm{~g}^{-1}\right.$ a $\left.1150 \mathrm{~m}^{2} \mathrm{~g}^{-1}\right)$.

Neste estudo a argila $P$ foi organofilizada pela troca cationica de íons de sódio por íons de fósforo. As argilas, antes e após a organofilização, foram avaliadas por fluorescência de raios-X (Tabela 1), observando-se que ocorreu uma redução da concentração dos cátions de cálcio, magnésio e, principalmente, de sódio, que variou de 3,27\% a $0,35 \%$, e um aumento na concentração de fósforo de $0,093 \%$ para $3,82 \%$ após o processo de organofilização. Esse resultado evidencia a eficiência da metodologia empregada na organofilização da argila.

A argila AO foi tratada com silano, GPTMS, visando a incorporação de anéis epóxidos a esse material. A silianização da argila 

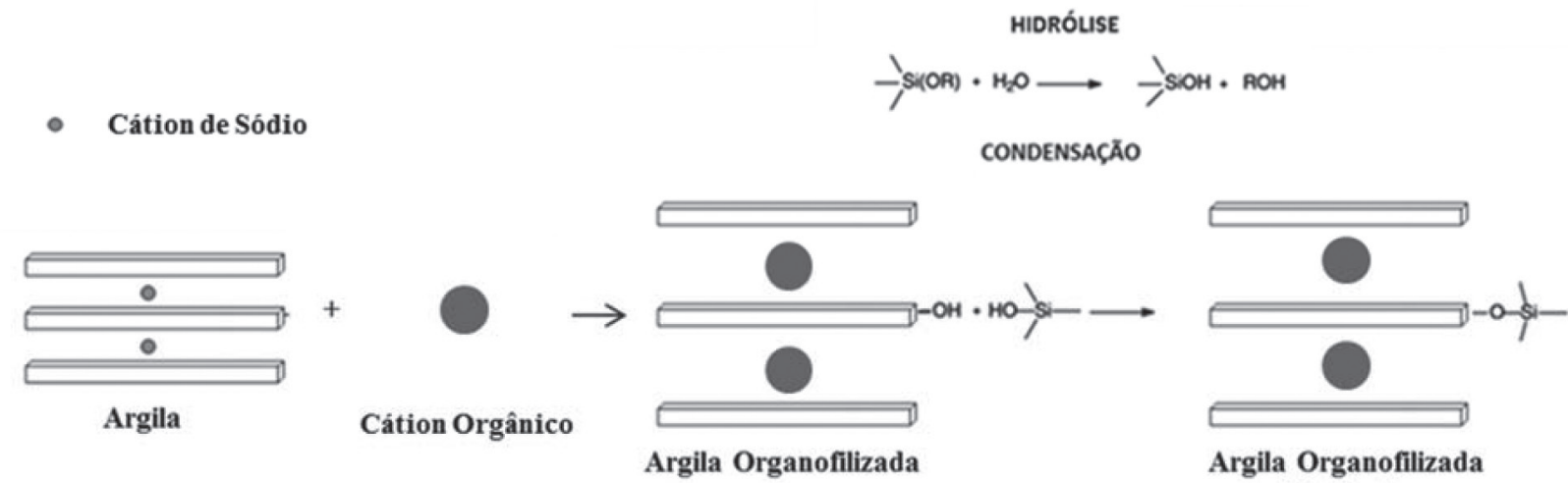

Argila Organofilizada Silanizada

Figura 2. Representação da organofilização e silanização da argila bentonita

Tabela 1. Composição química parcial da argila P e da AO obtida por XRF

\begin{tabular}{ccc}
\hline & Argila P (\% massa) & Argila AO (\% em massa) \\
\hline $\mathrm{Al}_{2} \mathrm{O}_{3}(\%)$ & 21,40 & 20,82 \\
$\mathrm{CaO}(\%)$ & 0,69 & 0,27 \\
$\mathrm{MgO}(\%)$ & 3,17 & 2,76 \\
$\mathrm{Na}_{2} \mathrm{O}(\%)$ & 3,27 & 0,35 \\
$\mathrm{P}_{2} \mathrm{O}_{5}(\%)$ & 0,09 & 3,82 \\
$\mathrm{SiO}_{2}(\%)$ & 66,04 & 66,06 \\
\hline
\end{tabular}

é interessante, pois possibilita a formação de ligações covalentes entre as partículas de argila silanizadas e moléculas orgânicas que possuam grupos funcionais reativos. Um esquema representativo da organofilização e da reação de silanização é mostrado na Figura 2. A silanização pode ocorrer nas superfícies internas ou externas e, também, nas arestas das lamelas da argila. . $4,26,40^{-}$

A Figura 3 apresenta os padrões de difração de raios- $X$ da argila natural, argila retida em peneira de $53 \mu \mathrm{m}$, argila $\mathrm{P}$, e das argilas AO e AOS, observando-se um espaçamento interlamelar basal (001) de $1,15 \mathrm{~nm}, 1,78 \mathrm{~nm}$ e $1,72 \mathrm{~nm}$, para as argilas $\mathrm{P}, \mathrm{AO}$ e AOS, respectivamente. Nota-se que a argila retida em peneira apresenta picos entre $20^{\circ}$ e $30^{\circ}$ devido à presença de quatzo, feldspato e clinoptilolite. ${ }^{5}$ $\mathrm{O}$ padrão de difração da argila $\mathrm{P}$ não apresenta picos entre $20^{\circ} \mathrm{e}$ $30^{\circ}$, indicando a retirada de feldspato e quartzo. Os demais picos não sofrem alteração após o peneiramento da argila. O aumento de $55 \%$ na distância interlamelar da $\mathrm{AO}$ em relação à argila $\mathrm{P}$ evidencia a intercalação do CTFF no espaço interlamelar da argila, como proposto na Figura 2, não causando alteração em sua organização estrutural. ${ }^{1,2,4,9,41}$ Já a silanização tem uma influência mínima na distancia basal, causando uma redução de cerca de $0,3 \%$, o que sugere que o silano se ligou principalmente aos grupos reativos presentes nas arestas e superfície da argila, o que pode ser explicado devido ao espaço interlamelar estar preenchido com o CTFF, dificultando a difusão do GPTMS entre as lamelas, como descrito pelos autores Chen et al. e Wang et al..$^{35,42}$ Esses autores relataram comportamento semelhante ao observado neste estudo ao silanizarem uma argila montmorilonita organofilizada. Wang et al. obtiveram valores de $1,2 \mathrm{~nm}$ para a argila montmorilonita, $1,9 \mathrm{~nm}$ para argila organofílica e $1,88 \mathrm{~nm}$ para a argila organofílica silanizada. ${ }^{35}$

Os espectros no infravermellho obtidos para a argila $\mathrm{P}, \mathrm{AO}$ e CTFF são mostrados na Figura 4, verificando-se alterações espectrométricas que evidenciam a presença do CTFF na AO, caracterizada pela presença das bandas em $750 \mathrm{~cm}^{-1}, 720 \mathrm{~cm}^{-1}$ e $690 \mathrm{~cm}^{-1}$ relativas ao grupo $\mathrm{CH}$ aromático do sal de fósforo, observa-se ainda a banda em $1436 \mathrm{~cm}^{-1}$ atribuída à ligação entre o fósforo e o anel aromático.

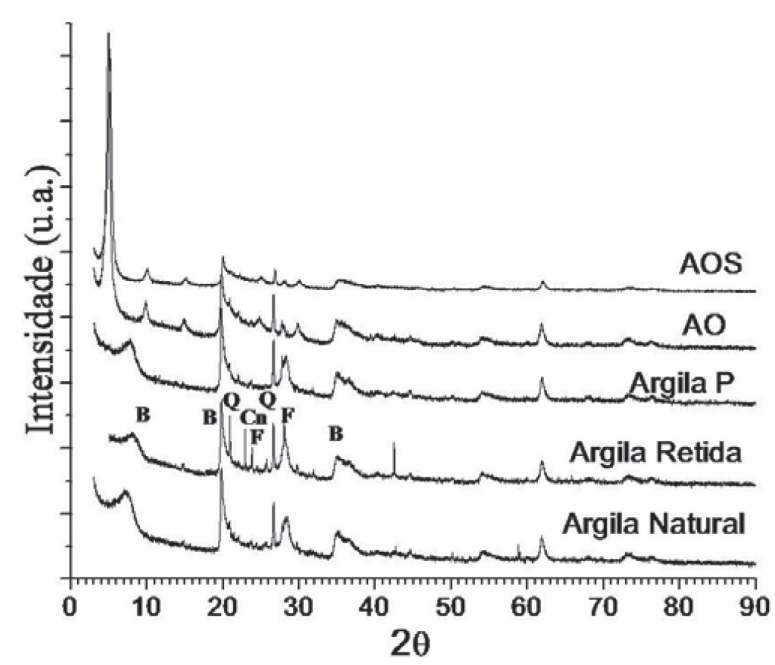

Figura 3. Difratogramas da Argila Natural, Argila Retida, Argila P, AO e AOS (B: Bentonita; Q: Quartzo; F: Feldspato; Cn: Clinoptilolite)

Também foi possível se observar um deslocamento da banda referente ao grupo Si-O de $994 \mathrm{~cm}^{-1}$ para $1015 \mathrm{~cm}^{-1}$ e o seu estreitamento. Estas alterações espectrométricas sugerem que a provável interação entre os grupos $\mathrm{Si}-\mathrm{OH}$ tenha sido enfraquecida pela presença do CTFF devido ao afastamento das lamelas da argila. Pode-se observar ainda uma redução das intensidades das bandas em $3421 \mathrm{~cm}^{-1}$ e $1633 \mathrm{~cm}^{-1}$, referentes ao estiramento e à deformação do grupo $\mathrm{OH}$, relativos à presença de umidade, caracterizando uma maior hidrofobicidade da $\mathrm{AO} .43,44$

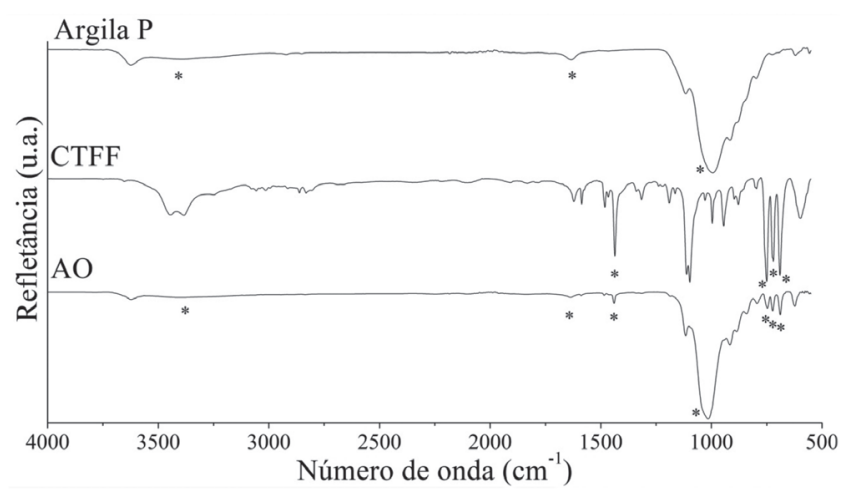

Figura 4. Espectros FT-IR (Reflexão - UATR) da Argila P, CTFF e AO

Na Figura 5, verificam-se alterações espectrométricas que evidenciam a presença do GPTMS na AOS, caracterizada pela presença 
Tabela 2. Porcentagens atômicas dos elementos químicos presentes nas argilas obtidas por XPS

\begin{tabular}{ccccccccc}
\hline \multirow{2}{*}{ Argila } & \multicolumn{4}{c}{ Composição superficial (\% atômica) } & \multicolumn{2}{c}{ Razão } \\
\cline { 2 - 9 } & $\mathrm{O} 1 \mathrm{~s}$ & $\mathrm{Si} 2 \mathrm{p}$ & $\mathrm{Al} 2 \mathrm{p}$ & $\mathrm{Na} 1 \mathrm{~s}$ & $\mathrm{C}$ 1s & $\mathrm{P} 2 \mathrm{p}$ & $\mathrm{Al} / \mathrm{Si}$ & $\mathrm{C} / \mathrm{Si}$ \\
\hline $\mathrm{P}$ & 40,1 & 18,9 & 6,2 & 0,55 & 2,8 & - & 0,33 & 0,15 \\
$\mathrm{AO}$ & 43,5 & 19,5 & 6,5 & - & 27,6 & 0,7 & 0,33 & 1,41 \\
AOS & 41,7 & 17,9 & 5,4 & - & 30,2 & 1,0 & 0,30 & 1,69 \\
\hline
\end{tabular}

das bandas em $2943 \mathrm{~cm}^{-1}$ e $2841 \mathrm{~cm}^{-1}$ relativas ao grupo $\mathrm{CH}$ do anel epóxido e $\mathrm{Si}-\mathrm{O}-\mathrm{CH}_{3}$ do GPTMS, respectivamente. Observa-se, ainda, o alargamento da banda na região de $1254 \mathrm{~cm}^{-1}$ relativa ao grupamento COC do anel epóxido do silano. ${ }^{44}$

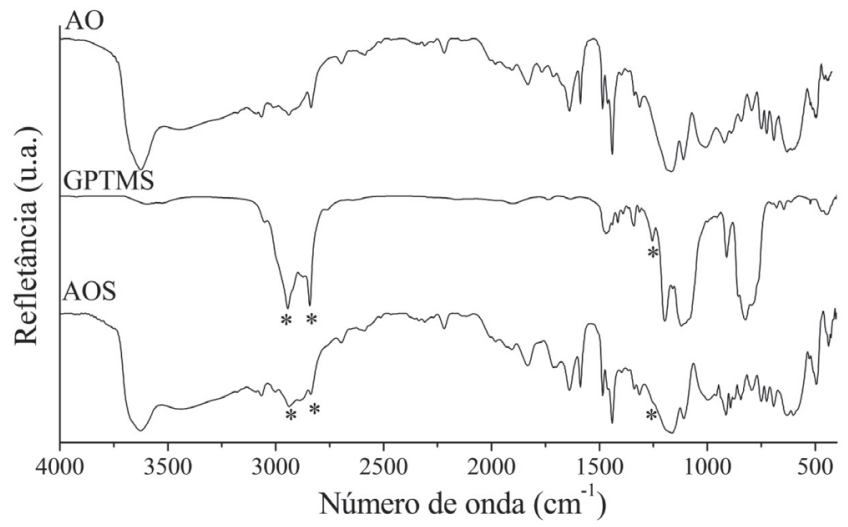

Figura 5. Espectros FT-IR (Reflexão - Drift) da AO, AOS e GPTMS

A superfície das argilas foi avaliada por XPS (Figura 6), sendo mostradas as porcentagens relativas dos diferentes elementos encontrados nas argilas na Tabela 2.

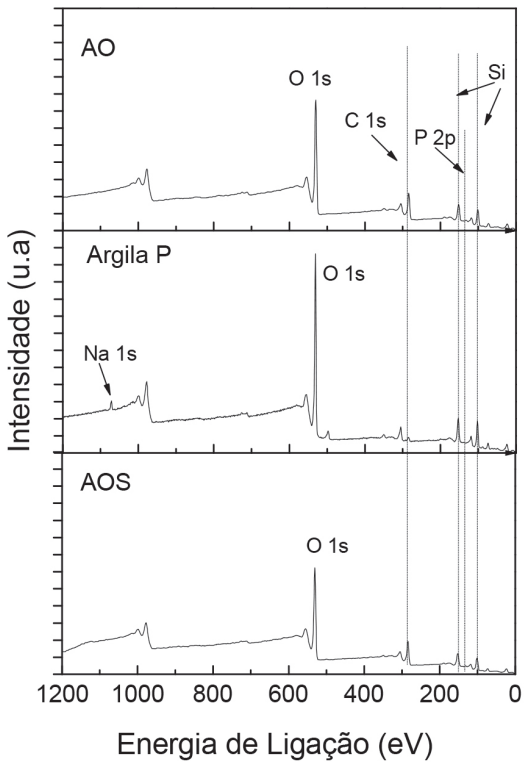

Figura 6. Espectros XPS da argila $P$ e argilas modificadas, $A O$ e AOS

O sódio, $\mathrm{Na}$ 1s (1072 eV), é identificado apenas na argila $\mathrm{P}$, como esperado, e o fósforo, $\mathrm{P} 2 \mathrm{p}(132,7 \mathrm{eV})$, é detectado em baixas quantidades nas argilas $\mathrm{AO}$ e AOS devido à substituição dos cátions de sódio por cátions de fósforo. Destaca-se que os teores de $\mathrm{Na} 1 \mathrm{~s}$ e P 2 p detectados por essa técnica são inferiores a $1 \%$, o que deve estar relacionado à profundidade superficial dessa análise, que varia de 2 a $5 \mathrm{~nm}$. Como esses cátions se encontram preferencialmente nas regiões interlamelares, a detecção desses elementos é baixa por XPS. O carbono, C 1s (285 eV), aparece em baixo teor na argila P devido à presença de $\mathrm{CO}_{2}$ adsorvido à sua superfície. ${ }^{31,45,46}$ Nas argilas modificadas o $\mathrm{C} 1 \mathrm{~s}$ é observado em maiores teores, o que pode ser explicado considerando-se as estruturas químicas do CTFF e do GPTMS (Figura 1) incorporados a essas argilas. Também a razão C/Si aumenta de acordo com argila $\mathrm{P}<\mathrm{AO}<\mathrm{AOS}$. De acordo com os resultados de espaçamento lamelar obtidos pela difração de raios X, o CTFF deve ocupar preferencialmente os espaços interlamelares, enquanto o GPTMS as arestas e a superfície da argila. A detecção do CTFF pela análise XPS deve-se principalmente ao fato da distância interlamelar ser da ordem de $1,78 \mathrm{~nm}$ na argila contendo CTFF, e a profundidade da análise XPS ser da ordem de $5 \mathrm{~nm}$. Com base na diferença das quantidades de $\mathrm{C}$ 1s encontradas nas argilas $\mathrm{AO}$ e AOS, pode-se inferir que o aumento na quantidade de $\mathrm{C} 1 \mathrm{~s}$ observado para a argila AOS é devido à ligação do GPTMS à supefície da argila organofilizada, podendo-se estimar um teor de silano incorporado à $\mathrm{AOS}$ entre 2-3\%. Quando se observa a variação da razão $\mathrm{Al} / \mathrm{Si}$, nota-se uma ligeira redução nesse valor para a argila AOS. De acordo com He et al., essa redução na identificação das placas octaédricas constituidas de $\mathrm{AlO}(\mathrm{OH})$ intercaladas entre duas placas tetraédricas $\mathrm{Si}-\mathrm{O}$ da argila pode estar associada à ligação dos grupos GPTMS à superfície da argila AOS, que interferem em sua identificação. ${ }^{45}$

A avaliação elementar das argilas foi realizada por meio da análise $\mathrm{CHN}$, sendo identificados diferentes teores de carbono e hidrogênio nas argilas submetidas aos diferentes tratamentos (Tabela 3). Conforme esperado, a presença de nitrogênio não foi detectada para estes materiais. O carbono representa 78,18\% do CTFF e $45,76 \%$ do GPTMS; a partir desses valores foi possível estimar a quantidade em massa de CTFF na AO $(20,9 \pm 0,1) \%$, e de GPTMS na $\operatorname{AOS}(3,2 \pm 0,8) \%$.

Tabela 3. Resultados obtidos pela análise $\mathrm{CHN}$ para as argilas

\begin{tabular}{ccc}
\hline & $\mathrm{C}(\%)$ & $\mathrm{H}(\%)$ \\
\hline Argila P & $0,05 \pm 0,01$ & $0,6 \pm 0,1$ \\
AO & $16,4 \pm 0,1$ & $1,1 \pm 0,2$ \\
AOS & $17,9 \pm 0,4$ & $1,4 \pm 0,4$ \\
\hline
\end{tabular}

Os perfis de decomposição térmica das argilas e dos compostos empregados em sua modificação são mostrados na Figura 7. As argilas $\mathrm{P}, \mathrm{AO}$, AOS e o CTFF apresentaram mais de uma perda de massa, sendo que a primeira variação de massa observada entre $25^{\circ} \mathrm{C}$ e $150^{\circ} \mathrm{C}$, é referente à liberação de água adsorvida tanto nas argilas como no CTFF. As variações devido à eliminação de água corresponderam à $(4,6 \pm 0,3) \%,(1,8 \pm 0,1) \%,(1,1 \pm 0,1) \%$ e $(2,6 \pm 0,1) \%$, para a argila $\mathrm{P}$, $\mathrm{AO}, \mathrm{AOS}$ e CTFF, respectivamente. Essa variação de massa foi menor para as argilas AO e AOS em relação à variação observada na argila $\mathrm{P}$, indicando uma maior hidrofobicidade das argilas modificadas..$^{29,47}$ A menor hidrofobicidade dessas argilas também foi observada pelos espectros no infravermelho desses materias (Figura 4). A decomposição térmica do CTFF ocorreu em um único estágio, entre $150{ }^{\circ} \mathrm{C}$ e $300^{\circ} \mathrm{C}$. O GPTMS mostrou decomposição térmica em uma única 


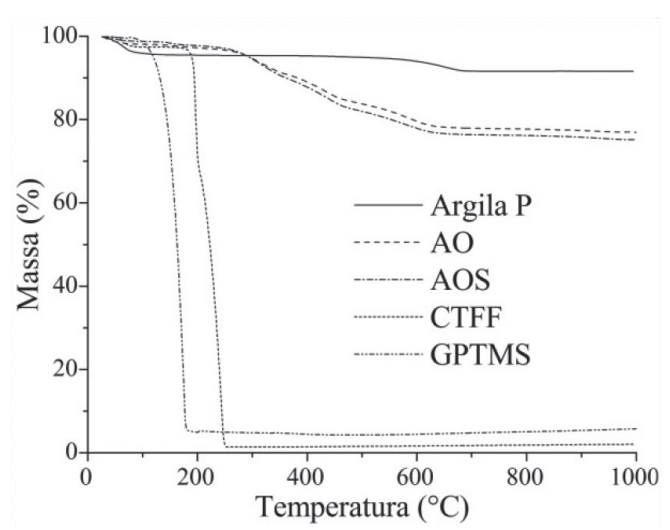

(a)

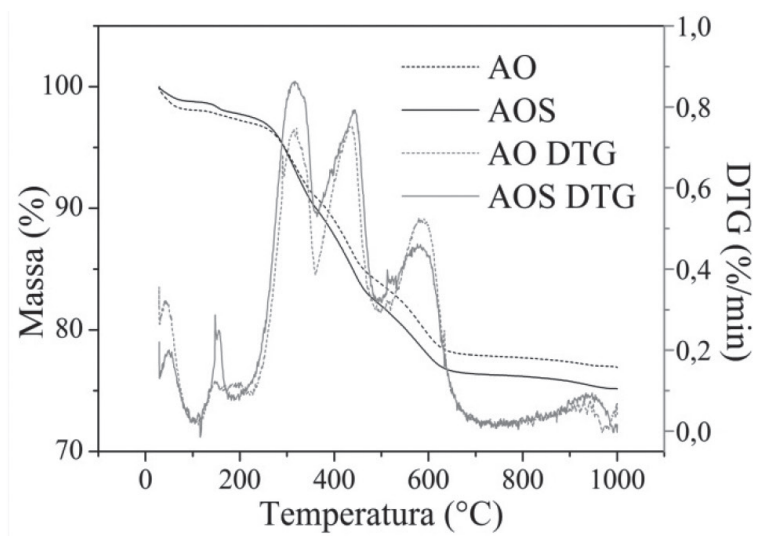

(b)

Figura 7. Curvas de variação de massa obtidas para Argila P, AO, AOS, CTFF E GPTMS (a) ampliação das curvas de variação de massa e as respectivas curvas derivadas obtidas para as argilas AO e AOS

etapa, ocorrendo até $200^{\circ} \mathrm{C}$, com variação de massa de $(94,8 \pm 0,3) \%$ referente à parte orgânica desse material.

$\mathrm{A}$ argila $\mathrm{P}$ apresentou a segunda perda de massa em uma faixa de temperatura maior que aquela observada para as argilas modificadas, ocorrendo entre $400{ }^{\circ} \mathrm{C}$ e $750{ }^{\circ} \mathrm{C}$, sendo de $(3,8 \pm 0,2) \%$. Essa variação de massa está relacionada à desidroxilação da argila. ${ }^{29,35}$

Em relação à decomposição térmica das argilas organolifizadas, na literatura são descritas temperaturas de ínicio de decomposição térmica entre $150{ }^{\circ} \mathrm{C}$ e $220{ }^{\circ} \mathrm{C}^{10,14,23,48} \mathrm{em}$ argilas para as quais a troca catiônica foi realizada com sais de amônio, enquanto argilas organofilizadas com sais de fósforo apresentam início de decomposição térmica entre $190{ }^{\circ} \mathrm{C}$ e $320^{\circ} \mathrm{C} . .^{10,23,48} \mathrm{~A}$ argila $\mathrm{AO}$ apresentou temperatura de decomposição térmica inicial em aproximadamente $220^{\circ} \mathrm{C}$, estando no ínicio da faixa de temperatura descrita para argilas organofilizadas com sais de fósforo. A variação de massa observada para as argilas AO e AOS entre $220{ }^{\circ} \mathrm{C}$ e $800{ }^{\circ} \mathrm{C}$ foi de $(19,2 \pm 0,3) \%$ para a $\mathrm{AO}$ e $(21,5 \pm 0,1) \%$ para a AOS. Essa variação está relacionada à desidroxilação da argila, como já mencionado para a argila $\mathrm{P}$ somada à decomposição térmica dos modificadores CTFF e GPTMS presentes nessas argilas. A variação de massa observada para a argila AOS foi maior em relação à observada no caso da argila $\mathrm{AO}$, caracterizando a presença do GPTMS nessa argila, que pode ser estimado em cerca de $2 \%$ a partir dessa diferença. Esse resultado é coincidente com os valores obtidos por CHN e XPS, que ficaram em torno de $3 \%$.

\section{CONCLUSÃO}

A organofilização da argila bentonita foi realizada com sucesso, sendo evidenciada pelo aumento no teor de fósforo e pela redução no teor de sódio quando comparadas a argila $\mathrm{AO}$ à argila $\mathrm{P}$ de acordo com os resultados de FRX. A varição no teor de carbono determinada por CNH e por XPS também confirmaram a substituição catiônica do sódio pelo sal quaternário de fósforo. A incorporação interlamelar do sal quaternário de fósforo na estrutura da argila causou um aumento de 55\% na distância interlamelar na $\mathrm{AO}$ em relação à argila $\mathrm{P}$, de acordo com os resultados de XRD.

As alterações espectrométricas observadas nos espectros FTIR indicaram a presença de CTFF na AO e sugeriram uma maior hidrofobicidade dessa argila em comparação à argila $\mathrm{P}$, que foi confirmada pela redução nos teores de umidade determinados por termogravimetria.

A presença de GPTMS na AOS foi evidenciada pelas alterações espectrométrica nos espectros no infravermelho e pelo aumento do teor de carbono determinado pelas técnicas de CHN e XPS. A redução da distância interlamelar de cerca de $0,3 \%$ causada pela silanização da argila organofilizada indicou que as moléculas do silano se ligaram preferencialmente à superfície e às arestas da argila. A metodologia empregada mostrou-se adequada na organofilização de argila do tipo bentonita sódica, permitindo sua incorporação em meios orgânicos diversos, tais como matrizes poliméricas utilizadas na obtenção de nanocompósitos ou em dispersões como tintas e recobrimentos.

\section{AGRADECIMENTOS}

À Coordenação de Aperfeiçoamento de Pessoal de Nível Superior (CAPES) pela bolsa concedida, e também a M. F. Diniz, M. F. P. Azevedo, A. Garcia, R. R. Cunha e M. A. M. de Souza pela assistencia técnica nas análises realizadas.

\section{REFERÊNCIAS}

1. Cavalcanti, J. V. F. L.; Abreu, C. A. M.; Sobrinho, M. A. M.; Baraúna, O. S.; Portela, L. A. P.; Quim. Nova 2009, 32, 2051.

2. Menezes, R. R.; Ávila Júnior, M. M.; Santana, L. N. L.; Neves, G. A.; Ferreira, H. C.; Cerâmica 2008, 54, 152.

3. Teixeira-Neto, E.; Teixeira-Neto, A. A.; Quim. Nova 2009, 32, 809.

4. Araújo, S. S.; Araújo, P. E. R.; Raposo, C. M.; Carvalho, L. H.; Silva, S. M. L.; Anais do Congresso Brasileiro de Engenharia e Ciência dos Materiais, Foz do Iguaçu, Brasil, 2006.

5. Önal, M.; Sarikaya,Y.; Alemdaroğlu, T.; Bozdoğan, I.; Turk. J. Chem. 2003, 27, 683.

6. Díaz, F. R. V.; Santos, P. S.; Quim. Nova 2001, 24, 345.

7. Coelho, A. C. V.; Santos, P. S.; Santos, H. S.; Quim. Nova 2007, 30, 1282.

8. Baltar, C. A. M.; Luz, A. B.; Insumos Minerais Para Perfuração De Poços De Petróleo, CTPETRO: Recife, 2003, cap. 2.

9. Ferreira, H. S.; Menezes, R. R.; Ferreira, H. S.; Martins, A. B.; Neves, G. A.; Ferreira, H. C.; Cerâmica 2008, 54, 77.

10. Hedley, C. B.; Yuan, G.; Theng, B. K. G.; App. Clay Sci. 2007, 35, 180.

11. Azeez, A. A.; Rhee, K. Y.; Park, S. J.; Hui, D.; Composites 2013, 45, 308.

12. Barbosa, R.; Araújo, E. M.; De Oliveira, A. D.; De Melo T. J. A.; Cerâmica 2006, 52, 264.

13. Xi, Y.; Frost R. L.; He, H.; Kloprogge, T.; Bostrom, T.; Langmuir 2005, 21,8675

14. Xie, W.; Gao, Z.; Pan, W. P.; Hunter, D.; Singh, A.; Vaia, R.; Chem. Mater. 2001, 13, 2979. 
15. Yeşilyurt, Z.; Boylu, F.; Çinku, K.; Esenli, F.; Çelik, M. S.; Appl. Clay Sci. 2014, 95, 176

16. Nguemtchouin, M. G. M.; Ngassoum, M. B.; Kamga, R.; Deabate, S.; Lagerge, S.; Gastaldi, E.; Chalier, P.; Cretin, M.; App. Clay Sci., 2015, 104, 110.

17. Dutta, A.; Singh, N.; Environ. Sci. Pollut. Res. 2015, 22, 3876.

18. Suin, S.; Shrivastava, N. K.; Maiti, S.; Khatua, B. B.; Eur. Polym. J. 2013, 49, 49.

19. Cui, L.; Bara, J. E.; Brun, Y.; Yoo, Y.; Yoon, P. J.; Paul, D. R.; Polymer 2009, 50, 2492.

20. Patel, H. A.; Somani, R. S.; Bajaj, H. C.; Jasra, R. V.; Appl. Clay Sci. 2007, 35, 194

21. Calderon, J. U.; Lennox, B.; Kamal, M. R.; Appl. Clay Sci. 2008, 40, 90.

22. Ollier, E.; Rodriguez, E.; Alvarez, V.; Composites, Part. A 2013, 48, 137.

23. Xie, W.; Xie, R.; Pan, W. P.; Hunter, D.; Koene, B.; Tan, L. S.; Vaia, R.; Chem. Mater. 2002, 14, 4837.

24. Gianni, A.; Amerio, E.; Monticelli, O.; Bongiovanni, R.; Appl. Clay Sci. 2008, 42, 116 .

25. Bertuoli, P. T.; Piazza, D.; Scienza, L.C.; Zattera, A. J.; Appl. Clay Sci. 2014, 87, 46.

26. He, H.; Tao, Q.; Zhu, J.; Yuan, P.; Shen, W.; Yang, S.; Appl. Clay Sci. 2013, 71, 15.

27. Qin, Z.; Yuan, P.; Zhu, J.; He, H.; Liu, D.; Yang, S.; Appl. Clay Sci. 2010, $50,546$.

28. Chen, G. X.; Yoon, J. S.; Polym. Degrad. Stab. 2005, 88, 206.

29. Qian, X.; Liao, M.; Zhang, W.; Polym. Int. 2007, 56, 399.

30. Vennerberg, D.; Rueger, Z.; Kessler, M. R.; Polymer 2014, 55, 1854.

31. Bugyi, L.; Oszk’o, A.; Solymosi, F.; Surf. Sci. 2000, 461, 177.
32. Kahr, G.; Madsen, F. T.; Appl. Clay Sci. 1995, 9, 327.

33. Yukselen, Y.; Kaya, A.; Eng. Geol. 2008, 102, 38.

34. http://www.sigmaaldrich.com, acessada em novembro 2017.

35. Wang, W. S.; Chen, H. S.; Wu, Y. W.; Tsai, T. Y.; Chen-Yang, Y. W.; Polymer 2008, 49, 4826.

36. Fonseca. M. G.; Airoldi, C.; Quim. Nova 2003, 5, 699.

37. Bergaya, F.; Theng, B. K. G.; Lagaly, G.; Handbook of clay science, $1^{\text {st }}$ ed., Elsevier: Oxford, 2006.

38. Hang, P. T.; Brindley, G.W. Clays Clay Miner. 1970, 18, 203.

39. Foletto, E.; Morgado, A. F.; Porto, L. M.; Anais do $44^{\circ}$ Congresso Brasileiro de Cerâmica, São Pedro, Brasil, 2000.

40. Spencer, M. W.; Hunter, D. L.; Knesek, B. W.; Paul D. R.; Polymer 2011, 52, 5369.

41. Rodrigues, A. W.; Brasileiro, M. I.; Araújo, W. D.; Araújo, E. M.; Neves, G. A.; Melo, T. J. A.; Polim.: Cienc. Tecnol. 2007, 17, 219.

42. Chen, G. X.; Kim, H. S.; Yoon, J. S.; Polym. Int. 2007, 56, 1159.

43. Bellamy, L. J.; The Infra-Red Spectra of Complex Molecules, $3^{\text {rd }}$ ed., Wiley: New York, 1975.

44. Smith, A. L.; Applied Infrared Sprectroscopy, Wiley: New York, 1979.

45. He, H.; Zhou, Q.; Frost, R. L.; Wood, B. J.; Duong, L. V.; Kloprogge, J. T.; Spectrochim. Acta, Part A 2007, 66, 1180.

46. Park, Y.; Ayoko, G. A.; Frost, R. L.; J. Colloid Interface Sci. 2011, 360, 440.

47. Djouani, F.; Herbst F.; Chehimi, M. M.; Benzarti, K.; Constr. Build. Mater. 2011, 25, 424.

48. Seyidoglu, T.; Yilmazer, U.; J. Thermoplast. Compos. Mater. 2015, 28, 86. 